\title{
General analytical model for distributed Brillouin sensors with sub-meter spatial resolution
}

\author{
Luc Thévenaz* and Jean-Charles Beugnot \\ EPFL Swiss Federal Institute of Technology, Institute of Electrical Engineering, Group for Fibre Optics, \\ STI IEL GR-SCI Station11, CH-1015 Lausanne, Switzerland
}

\begin{abstract}
A general analytic solution for Brillouin distributed fibre sensors with sub-meter spatial resolution has been obtained by solving the coupled wave equation by a perturbation method. The effect of the interaction of a square pump pulse with a continuous signal is described in full generality for all possible pumping schemes and for any detuning with the resonance condition of the Brillouin interaction. The model predicts how the acoustic wave, the signal amplitude and the gain spectral profile depend upon the pumping scheme. The analytical solution is an unprecedented tool to optimize the sensor configuration by determining the optimum pumping scheme.
\end{abstract}

Keywords: Fiber optics, stimulated Brillouin scattering, distributed fiber sensor, Brillouin fiber sensor, optical fiber sensor.

\section{INTRODUCTION}

During the past decade interesting observations and innovative configurations have been proposed to realize distributed measurements with sub-meter spatial resolution using stimulated Brillouin scattering in a pump-probe configuration. It was observed that, for pulses substantially shorter than the acoustic lifetime in silica, the measured gain profiles abandons their expected spectral broadening and the linewidth is gradually returning to the natural value given by the acoustic lifetime. This astonishing feature was first observed using pulses superimposed on a continuous pump level [1], then later using dark pulses [2] and eventually using $\pi$-phase pulses [3]. It was soon explained that this behaviour results from the sharp modified reflection of the pump wave on the pre-existing acoustic wave that is formerly built up by the presence of a continuous component in the pump wave [4]. The passage of very short pulses turns out to have a negligible effect on the acoustic wave amplitude and the changes in the signal amplitude are entirely dominated by the modified reflection of the pump wave on the steady acoustic wave during the pulse duration. Since the acoustic wave is essentially created by continuous waves, it will respond to frequency detuning according to the natural gain spectral profile.

These observations were theoretically all supported and justified by numerically solving the set of 3 coupled equations governing the interaction. The main problem arising from numerical justification is the absence of physical insight in the involved processes and their subsequent effect on the sensor response, and also the difficulty to optimize the different parameters of the sensor using an approach more efficient than a pure random choice that is gradually refined.

By a simple perturbation method and under realistic assumptions we could analytically solve the coupled equations governing the interactions for a totally general square pump pulse showing arbitrary complex amplitudes before, during and after the pulse. The general analytical solution expressed in the time domain matches the observed sensor response for all configurations proposed to date and for any pump-signal frequency detuning. Using this tool we can easily determine the sensor configuration providing the best response, and also anticipate and describe all unwanted contributions to the signal.

\section{ANALYTICAL MODEL}

The theoretical model is based on the set of 3 coupled equations governing the amplitudes of the 3 interacting waves:

$$
\frac{\partial A_{p}}{\partial z}+\frac{1}{V_{s}} \frac{\partial A_{p}}{\partial t}=i \frac{1}{2} g_{2} A_{s} Q
$$

$$
\frac{\partial A_{s}}{\partial z}-\frac{1}{V_{g}} \frac{\partial A_{s}}{\partial t}=-i \frac{1}{2} g_{2} A_{p} Q^{*}
$$

$$
\frac{\partial Q}{\partial t}+\Gamma_{A} Q=i g_{1} A_{p} A_{s}^{*}
$$

\footnotetext{
* luc.thevenaz@epfl.ch; phone +41 21693 4774; http://people.epfl.ch/luc.thevenaz
} 
where $A_{s}, A_{p}$ and $Q$ are the normalized envelopes of the signal, pump and acoustic amplitude, respectively. The frequency detuning parameter is $\Gamma_{A}=i\left(\Omega_{B}^{2}-\Omega^{2}-i \Omega \Gamma_{B}\right) / 2 \Omega$, where $\Omega_{B} / 2 \pi$ and $\Omega / 2 \pi$ are the Stokes resonance frequency and the pump-signal frequency difference, respectively, for a resonant interaction at a given point. The acoustic damping constant $\left(\Gamma_{B}\right)$ is related to the FWHM of the spontaneous Brillouin gain spectrum $\Delta v_{B}$ by $\Gamma_{B}=1 / \tau_{A}=\pi \Delta v_{B}$ where $\tau_{A}$ is the acoustic decay time equal to $11.8 \mathrm{~ns}$ in standard silica fibres. $g_{1,2}$ are the electrostrictive and elasto-optic coupling coefficients, respectively. In equation (3) the acoustic velocity is assumed to be much smaller than the light velocity $\left(V_{g}\right)$, so that the acoustic wave is considered as static and does not significantly propagate during the interaction. For a signal shorter than the acoustic lifetime, the transient analysis of the set of equation (1-3) is required. We used different approximations to determine a general analytical solution. First, the effect of the linear attenuation of light is neglected over the region where the waves interact. Second, the interaction is supposed weak enough that pump depletion can be neglected. Third, the only restriction imposed on the signal field is the small gain approximation during the interaction, which is well justified for an interaction of a few nanoseconds. Therefore, the signal amplitude $A_{s}(z, t)$ can be expressed as the sum of a continuous constant wave $A_{s}^{0}$ and a small varying term $a_{s}(z, t)$ resulting from the gain. The consequence is that $A_{s}$ can be considered as a constant in Eq. (1)-(3), except when $A_{s}$ is differentiated, as in the left term of Eq. (2) where only $a_{s}(z, t)$ is present. To calculate the solution let consider a very short segment of fibre extending from position $z_{0}$ to $z_{0}+\Delta z$ in which the interaction can take place, much shorter than the distance over which light propagates during the pump pulse length $T$ and the acoustic time $\tau_{A}$. The rest of the fibre is off resonance for stimulated Brillouin scattering (SBS) and no electrostriction is observed $\left(g_{1}=0\right)$. So, the interaction area is represented by a bounded distribution of the electrostriction $g_{1}(z)=g_{1}^{0}\left[u\left(z-z_{0}\right)-u\left(z-\Delta z-z_{0}\right)\right]$, where $u$ conventionally represents the unit step function. It must be pointed out that there is no loss of generality to consider an isolated interaction over one segment, since the system of equations subject to the approximations is linear in $g_{1}$. If the fibre is made of appended, but different, segments, the solution can be calculated separately for each segment (impulse response) and the total solution is simply given by the sum of each separated solution for $a_{s}$. The fact that the interaction is weak and the pump undepleted makes the pump propagation unaffected by the interaction and Equ. 1 can be

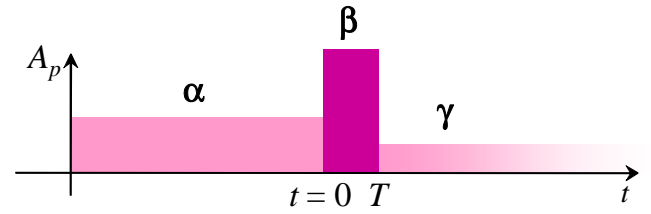

Fig. 1: Pump coding waveform where $\alpha$ is purely real and $\beta, \gamma$ are complex. The pump pulse duration $T$ defines the spatial resolution. discarded. The pump waveform $A_{p}(z, t)$ will thus be only function of the quantity $t-z / V_{g}$ and, as sketched in Fig. 1, the pump amplitude for a general square pulse can be represented by $A_{p}^{0}\left[\alpha+(\beta-\alpha) u\left(t-z / V_{g}\right)+(\gamma-\beta) u\left(t-T-z / V_{g}\right)\right]$, where $\alpha$ is a real dimensionless coefficient and $\beta, \gamma$ are complex.

Equ. (2-3) are solved in the Laplace domain and the following general solution for the signal amplitude in the time domain is obtained for a very short interaction segment $\Delta z$ (impulse response):

$a_{\mathrm{s}}(z, t)=-\frac{g}{\Gamma_{A}}\left\{\alpha^{2}-\left.\alpha^{2}\right|_{i \geq t_{0}}+\left.\left(\beta \beta^{*}-\beta\left(\beta^{*}-\alpha\right) \mathrm{e}^{-\Gamma_{A}\left(t-\frac{2 z_{0}-z}{V_{s}}\right)}\right)\right|_{t_{0}+T \geq t \geq t_{0}}+\left.\left(\gamma^{-}-\left[\gamma \beta^{*}\left(1-\mathrm{e}^{\Gamma_{A} T}\right)-\gamma\left(\alpha-\gamma^{*} \mathrm{e}^{\Gamma_{A} T}\right)\right] \mathrm{e}^{-\Gamma_{A}\left(t-\frac{2 z_{0}-z}{V_{B}}\right)}\right)\right|_{t \geq t_{0}+T}\right\}$

where $g$ is a gain factor equal to $g_{1}^{0} g_{2} I_{p}^{o} A_{s}^{0} / 2 \Gamma_{A}$.

This expression is clearly made of three different terms, each corresponding to instances in the pump pulse sequence. First a constant gain is observed prior to the presence of the pulse, represented by the factor $\alpha^{2}-\left.\alpha^{2}\right|_{t \geq t_{0}}$. Then, the interaction starts with the onset of the second term that manifests as an abrupt signal amplitude change, identified as the first Brillouin echo, that slowly decays exponentially during the pump pulse duration $T$. When the pump pulse ends, the second term stops contributing and the third term takes over as another step amplitude change - the second Brillouin echo - that shows the same slow exponential decay. Note that for $\gamma=0$, the third term vanishes. 


\section{DISCUSSIONS}

The validity of the solution explicited in expression (4) has been tested by comparing the Stokes signal amplification in different pumping configuration. The first test was performed on a pure bright pulse configuration with a zero background, so that $\alpha=\gamma=0$ and $\beta=1$. The time instance when the pulse enters the fiber was arbitrarily set to $10 \mathrm{~ns}$ and the Brillouin resonance frequency was fixed at $11 \mathrm{GHz}$. The red line in Fig. 2(a) represents the bright pulse spectrum for a pump pulse of $1 \mathrm{~ns}$. We observe in this case the classic response of a widely broadened spectral profile for short pump pulses. A solution to suppress this broadening is to pre-activate the acoustic wave before the pulse passage [4]. The next test was performed for the same pump pulse conditions, but with a non-zero background. The spectra plotted in Fig. 2 represent the step change of the signal amplitude as a function of detuning and for different fractional backgrounds. The presence of a continuous component activates the acoustic wave before the pulse passage. Then, the gain gradually increases during the pulse duration.
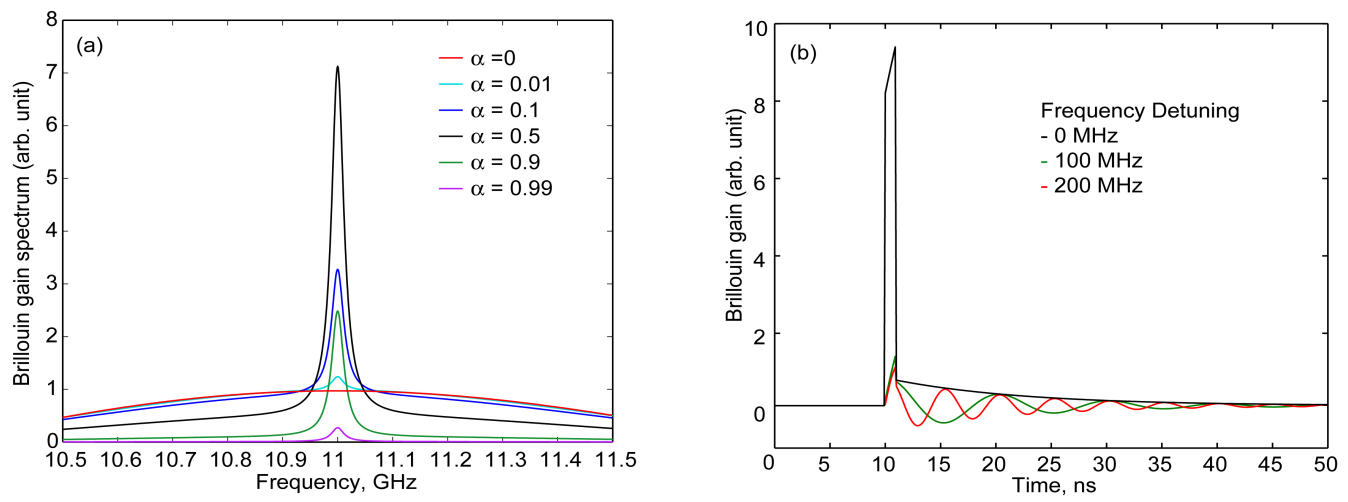

Fig. 2 : (a) Calculated Brillouin gain spectrum in a bright pulse configuration $(\beta=1)$ for different CW background. (b) Impulse response in the time domain at different frequency detuning for a $1 \mathrm{~ns}$ bright pulse with optimal CW component $(\alpha=\gamma=0.5, \beta=1)$.

In the case of very low backgrounds ( $\alpha=0.1$, see Fig. 2(a)) we observe a pedestal in the Brillouin spectrum. On the contrary, for very large $\mathrm{CW}$ components $(\alpha=0.9)$ the pedestal vanishes but the gain contrast is very low. The contrast is function of the difference between the amplitude during the pump pulse $(\beta)$ and the background amplitude before the interaction $(\alpha)$. The most efficient configuration can be calculated and corresponds to $\alpha=\gamma=0.5$ and $\beta=1$. We represent in Fig.2 (b) the impulse response of the signal change in the time domain for the most efficient bright pulse configuration at different frequency detuning. Off resonance, we clearly observe an oscillation on the signal amplitude after the pulse passage. Considering the case of a non-zero detuning in the Brillouin gain process, we add to the damping coefficient $\left(\Gamma_{A}\right)$ an imaginary part whose magnitude corresponds to the detuning $\Omega_{\mathrm{B}}-\Omega$, where $\Omega$ is the frequency difference between pump and signal. This introduces an oscillatory term into the acoustic wave equation (eq. 3), and ultimately gives rise to an oscillating response on the signal. In figure 2 (b), it can be seen that the oscillation frequency corresponds to the frequency detuning.

In the dark pulse configuration [2], the pump is turned off for a time interval $T$, so that no interaction is present and the constant background amplification is stopped. The acoustic wave keeps an inertial vibration during the dark pulse duration and the background amplification is restored when the pump light is turned on again. In Fig. 3, we observe that the contrast in this configuration is twice larger than for the bright pulse with an optimal background. However, a high contrast is necessary for ultimate spatial resolutions and it is primarily given by $\alpha(\beta-\alpha)$, as deduced from Equ. 4. A $\pi$ phase pulse modulating a $\mathrm{CW}$ pump wave

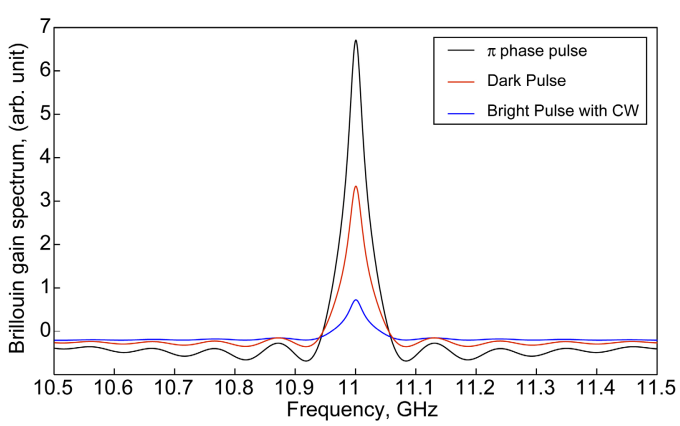

Fig. 3: Calculated Brillouin gain spectrum in three different pump pulse configuration for $T=1 \mathrm{~ns}$. Black line, $\pi$ phase pulse $(\alpha=\gamma=1, \beta=-1)$; red line, dark pulse $(\alpha=\gamma=1, \beta=0)$ and blue line, bright pulse with background $(\alpha=\gamma=0.5, \beta=1)$. The maximum contrast at Brillouin resonance in the different configuration is clearly visible. 
creates a maximum amplitude difference at the pulse edges. When a $\pi$ phase pulse is applied at $t=10 \mathrm{~ns}$, an abrupt amplitude change is observed that slowly decays exponentially during the pulse interval $T$, as shown in Fig. 4(a). This represents the sudden phase change of the pump that contributes destructively on the signal when reflected by the acoustic wave and the subsequent slow phase change of the acoustic wave to adapt to the new phase situation between pump and signal (first echo). As shown in Equ. 4, this term stops contributing when the pump pulse ends at $t=11 \mathrm{~ns}$ and the third term takes over. This manifests as another abrupt amplitude change when the pump phase suddenly jumps, and the same slow exponential decay is observed while the acoustic wave gradually adapts to the novel phase situation. This second echo is proportionally smaller for shorter pulses and turns negligible for vanishingly short pulses. In the classical case of a zero background bright pulse, the interaction between pump and signal starts at $\mathrm{t}=10 \mathrm{~ns}$ and we observe a slow exponential gain. After the pulse passage at $\mathrm{t}=11 \mathrm{~ns}$, the pump is turned off and the interaction stops brutally. In the case of short interactions $\left(T<\tau_{A}\right)$, the low gain actually results from the inertial behaviour of the acoustic wave, with a time constant given by the acoustic lifetime $\tau_{A} \cong 10 \mathrm{~ns}$.
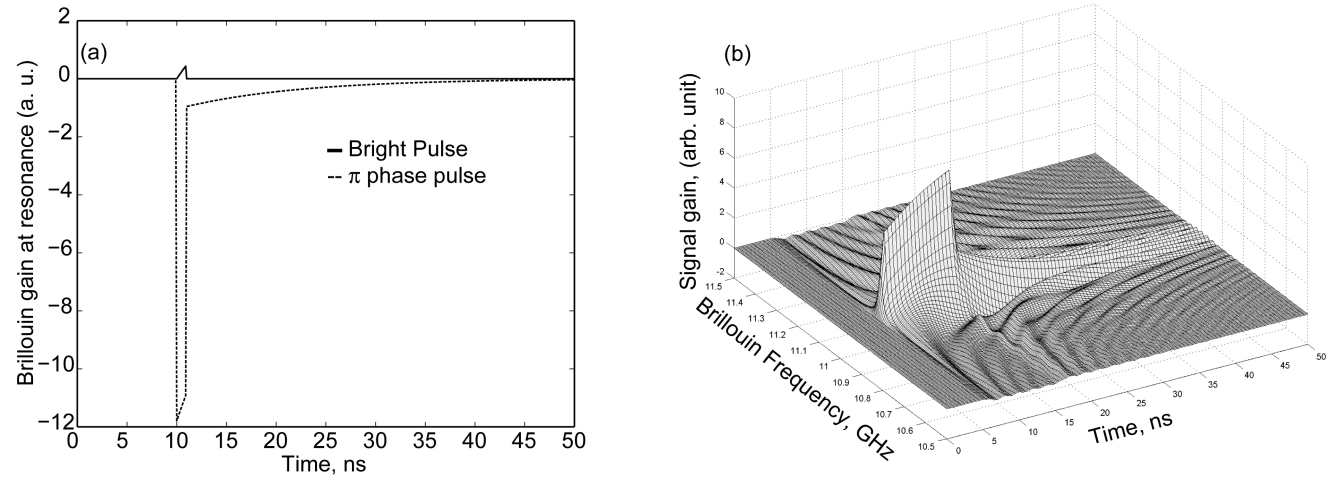

Fig. 3: (a) Calculated impulse response of the signal gain in the case of a zero background bright pulse $(\alpha=\gamma=0, \beta=1)$ and a $\pi$ phase pulse $(\alpha=\gamma=0, \beta=-1)$ for $T=1 \mathrm{~ns}$. In the case of a $\pi$ phase pulse, the gain in 27 times larger than for a bright pulse. The 2 nd echo is clearly observed at $t=t_{0}+T$. (b) Calculated 3D signal amplitude diagram for a $1 \mathrm{~ns} \pi$ phase pulse as a function of distance and frequency for a an interacting fibre segment of finite length $L=1 \mathrm{~m}$.

The study in the time domain clearly shows that the inertial response of the acoustic wave is a penalty in the classical zero background bright pulse configuration by preventing the acoustic wave from building up during a short pump pulse. On the contrary, in a configuration based on the Brillouin echoes, this inertial response is clearly an asset since we take an advantage of the slow decay of the acoustic wave. A longer acoustic lifetime will even lead to a better response, by reducing the importance of the $2^{\text {nd }}$ echo. In Fig. 4(b), the analytical solution is used to calculate the 3D signal amplitude for a $\pi$ phase pump pulse of $1 \mathrm{~ns}$ on a $1 \mathrm{~m}$ fiber length, to describe a realistic measurement. We clearly see the two sharp amplitude change at $t=10 \mathrm{~ns}$ and $t=20 \mathrm{~ns}$ corresponding to the interaction area, and the background off- resonance oscillations. This diagram is obtained instantaneously without numerical integration and the comparison with real measurements shows a perfect matching. The full knowledge of the time domain response of a given distributed sensor configuration based on the Brillouin echoes (BEDS) is decisively helpful to deconvolve the interfering effect of the second echo that may seriously screen the real Brillouin response of the system.

The authors would like to acknowledge the support from Omnisens SA, the tight collaboration with ETHZ-IGT and the helpful discussions and support from Stella Foaleng Mafang and from Tom Sperber visiting from Tel Aviv University.

\section{REFERENCES}

1. X. Bao, A. Brown, M. DeMerchant, and J. Smith, "Characterization of the Brillouin-loss spectrum of single-mode fibers by use of very short (<10-ns) pulses", Optics Letters, 24, p.510 (1999).

2. A. W. Brown, B. G. Colpitts, and K. Brown, "Distributed Sensor Based on Dark-Pulse Brillouin Scattering", IEEE Photonics Technology Letters, 17, p.1501 (2005).

3. L. Thévenaz, and S. Foaleng Mafang, "Distributed fiber sensing using echoes", Proceedings of $19^{\text {th }}$ International Conference of Fiber Sensors, (SPIE, Perth, WA, Australia), Vol 7004, (2008).

4. V. Lecoeuche, D.J. Webb, C.N. Pannell, and D.A Jackson, "Transient response in high-resolution Brillouin-based distributed sensing using probe pulses shorter than the acoustic relaxation time", Optics Letters, 25, p.156-158, (2000) 